Slightly revised for Revue française de Sociologie, 2004, 45(2) : 339-366

\title{
Contrainte et liberté dans le travail de conception architecturale
}

\author{
Dominique Raynaud ${ }^{1}$
}

Résumé : Cet article tente de clarifier les concepts de liberté et de contrainte à partir d'une étude de la conception architecturale, activité que les praticiens disent être un espace contraignant tout en parvenant à concevoir des solutions inédites. L'étude vise des résultats factuels et théoriques. Factuels, car il apparaît que les difficultés de l'exercice professionnel ne résultent pas tant des contraintes que de la sur-responsabilisation et de la sous-faisabilité des études qu'elles induisent. Théoriques, car les faits plaident pour une révision fondamentale du rapport négatif par lequel se définissent habituellement la liberté et la contrainte. Ces faits sont par exemple que les interdictions sont moins contraignantes que les prescriptions et que certaines stratégies de « phagocytage » peuvent trouver la voie de la liberté dans le respect à la lettre d'une contrainte.

Mots-clefs : contrainte, liberté, travail de conception, architecture.

Summary: This paper attempts to clarify the concepts of freedom and constraint through a study of architectural design work, an activity that its practitioners represent as a constraining space, though they in fact manage to find new solutions within. The study aims at both factual and theoretical results: factual in that the difficulties of exercising this profession seem to be due less to constraints than the excessive degree of responsability and near impracticability of the studies they induce; theoretical because certain facts suggest that a fundamental revision of the negative relation generally understood to obtain betwwen freedom and constraint is in order. Two examples of such facts: prohibitions are not as constraining as prescriptions, and certain 'absorption' strategies can open a path to freedom while following a given constraint 'to the letter'.

Keywords: constraint, freedom, design tasks, architecture.

\section{Introduction}

Alors que Les architectes, métamorphoses d'une profession libérale faisaient de la conception le noyau dur de l'expertise professionnelle de l'architecte (Moulin et al., 1973 ; Moulin et Lautman, 1978), les récents développements de la sociologie de l'architecture (Toussaint et Younès, 1997 ; Tapie, 1999 ; Champy, 1999 ; etc.) insistent plutôt sur le caractère incertain

${ }^{1}$ Université Pierre-Mendès-France, BP 47, 38040 Grenoble cedex 9 ; Laboratoire d'architecturologie et de recherches épistémologiques sur l'architecture (Paris); dominique.raynaud@upmf-grenoble.fr 
de cette définition. Champy observe par exemple que, « À l'inverse des médecins ou des hommes de loi, qui fournissent les exemples les plus typiques des professionnalisations réussies, les architectes n'ont pas réussi à imposer une définition univoque et stable des compétences sur lesquelles fonder leur professionnalisation » (1999 : 35). Cette mise en cause tient au fait que la conception se déroule dans un contexte plus complexe que jamais, en raison du nombre croissant d'acteurs participant au projet ${ }^{2}$. Cette prolifération des acteurs est elle-même un effet du cadre réglementaire de plus en plus coercitif de la conception. Plus le niveau des exigences extérieures est élevé, moins l'architecte généraliste est en mesure de répondre aux attentes spécifiques de ses interlocuteurs, et plus il est contraint de s'entourer de spécialistes auxquels il doit déléguer des parties de la mission de conception. Une étude de l'évolution de la profession d'architecte depuis les années 1970 devrait reposer, non tant sur le constat approfondi de l'érosion des pouvoirs de l'architecte que sur les raisons fondamentales de cette érosion, que bon nombre de praticiens disent être le cadre d'exercice de plus en plus contraignant de la profession.

Cet article se propose d'étudier comment l'architecte parvient à ouvrir un espace de liberté dans une situation de contraintes multiples. Une telle étude est à même d'apporter deux types de résultats. Des résultats factuels, tout d'abord, en ce qu'elle peut clarifier le statut de la liberté de l'architecte : il n'est pas sûr que toutes les injonctions adressées au concepteur exercent la même contrainte sur lui, ni que la contrainte soit exercée par le contenu même de l'injonction (elle peut aussi résulter du travail contextuel qu'elle induit). Des résultats théoriques, ensuite, car si la liberté résulte apparemment des stratégies mises en œuvres pour tourner licitement la contrainte, il n'est pas sûr que toutes les stratégies se conforment au rapport négatif liberté/contrainte envisagé par la définition classique, repris par l'analyse stratégique. Nous entendons mener l'étude sur deux fronts : acquérir les résultats factuels sur le front des pratiques professionnelles et du droit de l'architecture; les résultats théoriques sur le front de la sociologie et de la philosophie de l'action. Telles seront les deux orientations de cette étude.

\footnotetext{
${ }^{2}$ Moulin et Lautman voyaient dans « l'éclatement et la fragmentation de la mission traditionnelle répartie en une pluralité d'acteurs concurrents » (1978 : 50) un signe de déclin de la profession d'architecte. L'hypothèse d'une tendance durable à l'éclatement de la mission globale de conception et, par suite, à la multiplication des « ingénieries spécifiques » (Prost, 1999) semble aujourd'hui se confirmer.
} 


\section{Liberté et contrainte}

Commençons par poser quelques termes avec un minimum de rigueur. Distinguons la forme de la contrainte de son contenu, en réservant le terme d'injonction au contenu objectif de celle-ci, le terme de contrainte à l'effet qu'exerce un individu sur un autre individu, au moyen de cette injonction. L'injonction se décline en : ordre, si elle a pour effet que quelque chose soit fait; permission, si elle a pour effet que quelque chose puisse être fait ; interdiction, si elle a pour effet que quelque chose ne soit pas fait. Ces définitions sont un patrimoine commun de la philosophie et de la sociologie, focalisée depuis Simmel et Weber ${ }^{3}$ sur l'examen des « actions réciproques » [Wechselwirkungen].

C'est probablement en ayant à l'esprit des oppositions telles que : homme libre/esclave, homme libre/détenu, raison/passions, etc. que la philosophie morale classique a donné de la liberté une définition négative qui l'oppose à la contrainte (et qui coïncide avec la définition de sens commun de ces notions). Moritz Schlick écrit par exemple : « La liberté est le contraire de la contrainte, l'être humain est libre lorsqu'il n'est pas soumis à une contrainte » (1997 : 31). La sociologie de l'action et la philosophie de l'action sont à l'origine de deux révisions (respectivement faible et forte) de cette première définition.

\subsection{Première révision}

On doit à la sociologie, et en particulier à l'analyse stratégique, d'avoir entrepris une première révision de la définition classique. Dans L'acteur et le système, Crozier et Friedberg ${ }^{4}$ (1977) décrivent le jeu entre liberté des acteurs et contraintes structurelles en ces termes :

${ }^{3}$ Weber, dans l'étude des formes de l'ordre légitime, distingue entre convention et droit : « [Konvention], lorsque sa validité est garantie extérieurement par la chance que, si l'on s'en écarte à l'intérieur d'un groupe d'hommes déterminé, on s'expose à une réprobation [...]; Droit [Recht], lorsque la validité est garantie extérieurement par la chance d'une contrainte (physique ou psychique), grâce à l'activité d'une instance humaine, spécialement instituée à cet effet, qui force au respect de l'ordre et châtie la violation. » (1971, 1 : 68). Nous conservons le terme de « contrainte » pour désigner l'effet de la menace avant le déclenchement de la sanction.

${ }^{4}$ Même si l'architecte n'agit pas dans un univers à caractère purement bureaucratique, les règles du métier sont assez stables et les contraintes de conception suffisamment codifiées pour s'inspirer du cadre défini par Crozier et Friedberg (1977), étendu à de nouvelles situations par Friedberg (1992, 1993). 
« [La conduite humaine] est toujours l'expression et la mise en œuvre d'une liberté, si minime soit-elle. Elle traduit un choix à travers lequel l'acteur se saisit des opportunités qui s'offrent à lui dans le cadre des contraintes qui sont les siennes [...] Une situation organisationnelle donnée ne contraint jamais totalement un acteur. Celuici garde toujours une marge de liberté et de négociation. Grâce à cette marge de liberté [...] chaque acteur dispose ainsi de pouvoir sur les autres acteurs » $(1977: 45-46,91)$.

Selon cette conception : 1) l'action des individus est contrainte par le système social ; 2) l'individu garde une part de liberté ; 3) celle-ci résulte d'une construction active par l'acteur qui repousse, contourne, etc. les contraintes de la situation dans laquelle il agit. La révision (faible) opérée par Crozier et Friedberg (1977) tient à ce que :

- elle insiste sur le fait que liberté et contrainte modèlent simultanément l'action réelle, alors que la définition classique insistait sur l'alternative : liberté ou contrainte (sur le modèle : homme libre ou esclave).

- elle reprend à la philosophie classique sa définition négative de la liberté (les contraintes du système sont bien le contraire de la liberté individuelle) en considérant que les contraintes sont au fond des conditions de l'activité libre.

\subsection{Deuxième révision}

La philosophie analytique de l'action contemporaine a proposé une deuxième révision à partir d'une relecture des notes de Wittgenstein sur le fait d'« obéir à une règle » ${ }^{5}$. De façon générale, les recherches actuelles ont « désarticulé » les concepts de liberté et de contrainte (Melden, 1961, Chisholm [1964], Nozick, [1969], Neuberg 1990). Chisholm, corrigeant une formule de Melden, propose de reconnaître l'existence d'une liberté quand on peut dire d'un individu :

[1] « Il eût pu choisir d'agir autrement » (Chisholm, $1997: 43$ ).

La révision du concept de liberté est alors plus radicale que celle envisagée par l'analyse stratégique. Neuberg (1990) a montré que les théories classiques de la contrainte, d'Aristote à Ricoeur, se mettent en défaut en invoquant des exemples n'illustrant pas le rapport entre

${ }^{5}$ Cf. Philosophische Untersuchungen, §§ 199-208 (Wittgenstein, 1961 : 201-204). 
liberté et contrainte, mais le rapport entre action et mouvement involontaire. On ne peut parler de contrainte, conclut l'auteur, « que si le sujet est exposé à une menace de la part d'autrui » (1990 : 494). Il se fonde sur les travaux de Nozick [1969] qui énoncent des critères de contrainte dont les plus importants sont :

[2] « $\mathrm{P}$ menace de faire une certaine chose au cas où $\mathrm{Q}$ ferait $\mathrm{A}$ (et $\mathrm{P}$ sait qu'il profère cette menace) »,

[3] « Du fait de cette menace, accomplir A devient sensiblement moins désirable pour Q que de ne pas l'accomplir $[\ldots]^{6} »$ (Nozick, $\left.1991: 273\right)$.

C'est la menace d'une sanction ${ }^{7}$ et l'intérêt qu'un individu peut avoir de s'épargner cette sanction $^{8}$ qui fonde la définition contemporaine de la contrainte. La révision (forte) opérée par la philosophie analytique de l'action tient à ce que :

- elle identifie une contrainte là où un individu pense être exposé à une contrainte. Neuberg écrit : «Il n’est pas nécessaire qu'il y ait réellement une tentative de contrainte de la part de P. Il faut (et il suffit) que Q le croie » (1990: 498).

— elle rejette la définition négative de la liberté comme opposé de la contrainte. Neuberg souligne par exemple que « La contrainte n'abolit pas, en général, la décision et le choix » (1990 : 518).

${ }^{6}$ Les quatre autres sont : «P profère cette menace pour amener Q à ne pas accomplir A et avec l'intention que Q se rende compte de cette menace; Q ne fait pas A ; Les paroles ou les actes de P font partie des raisons pour lesquelles Q ne fait pas A ; Q sait que $\mathrm{P}$ a menacé de faire la chose mentionnée dans la condition (1), au cas où il accomplirait A. » (Nozick, 1991 : 273). Nous ne retiendrons ici que les deux premiers critères consensuels. Dans le débat actuel, les autres conditions sont jugées tantôt insuffisantes, tantôt superflues. Ainsi, le sixième critère est ajouté par Nozick pour distinguer contrainte et avertissement, mais Neuberg (1990) montre que l'avertissement, en ce qu'il constitue une menace préventive, exerce aussi une contrainte.

${ }^{7}$ « La sanction peut être définie comme une menace, explicite ou implicite, de punition pour avoir désobéi à la norme. L'existence d'une menace de punition n'est pas, par elle-même, un motif d'obéissance. La crainte de la punition en est un, cependant. » (von Wright, 1963 : 125). Ce rapport de la contrainte à la sanction est perçu par Schlick : «Les prescriptions s'opposent souvent aux désirs naturels des gens [...] ce qui entraîne que certains, tout en s'y conformant, les vivent comme des contraintes. C'est par des sanctions (peines) que l'État oblige alors les citoyens à mettre leurs désirs en accord avec les exigences de la loi. » (1997: 30).

${ }^{8}$ Ce qui rapproche la sociologie et la philosophie. François Chazel note : « L’intérêt bien compris de l'acteur l'amène à tenir compte des risques de sanction négative dans ses calculs : le respect de la norme lui apparaitrait comme la voie la plus sûre. Le contrôle social prendrait ici une forme négative, celle de la contrainte 
On touche là aux limites de la définition de l'action sociale comme choix exercé dans un environnement de contraintes : car les contraintes ne constituent même plus le « cadre » ou les « conditions » de l'activité libre. Cette redéfinition résulte d’une conception inédite de la contrainte, désormais affranchie de toute référence négative à la notion de liberté. L'un des objectifs théoriques de cette étude sera de savoir s'il existe des chances d'introduire cette révision radicale en sociologie.

\subsection{Point de vue des praticiens}

Les architectes n’ont guère pris appui sur ces révisions successives : la profession fait toujours l'objet de descriptions antagonistes et certains praticiens continuent de dichotomiser leur statut en se percevant tantôt comme de purs artistes ${ }^{9}$, tantôt comme des techniciens chargés de traduire en espace des volontés extérieures qui leur échappent ${ }^{10}$. Quelques uns articulent contrainte et liberté. Ces positions se retrouvent parmi les architectes interrogés ${ }^{11}$ :

« La phase de conception, c'est un lieu de liberté où je m'amuse à trouver des solutions. C'est le fondement même du travail de l'architecte. C'est vrai que je suis parti dans mon métier avec cette idée-là, et je l'ai gardée pratiquement jusqu'à aujourd'hui. »

externe. » $(1990: 451)$.

${ }^{9}$ L'architecte est un «poète », un « rêveur de formes » (Montlibert, $1995: 47$ ).

${ }^{10}$ «L'architecte ne peut rien faire aujourd'hui... Il n’y a plus de projet, c'est un corset dans lequel on ne peut plus lever le petit doigt » (architecte in Moulin, 1973 : 44). « La confrontation aux contraintes externes transforme la nature du travail de concepteur. [L'architecte] n'apparaît plus comme un artiste libre de donner les pleins pouvoir à sa fantaisie ou à son imagination créatrice » (Farel, 1995 : 53).

${ }^{11}$ La présente étude repose sur des données composites que l'on peut catégoriser de la façon suivante : 1) Un dépouillement thématique des revues professionnelles durant les dix dernières années (auquel nous avons ajouté les références citées par les architectes interrogés) ; 2) Un dépouillement thématique du Code de l'urbanisme et du Code de la construction (mise-à-jour 2000) ; 3) Cinq entretiens semi-directifs sur la perception et l'action des concepteurs face aux contraintes. L'étude visant à identifier les différentes stratégies d'action des architectes, nous avons adopté le principe d'un « comparatisme divergent » (Raynaud, 1998 : 36-41, 2002 : 149-151), qui consiste à supposer que la divergence des réponses obtenues est fonction de la différence des situations individuelles. Le nombre d'entretiens est alors réduit par la présélection d'acteurs placés dans des situations différentes : architecte jeune vs âgé, architecte libéral vs salarié, exerçant dans une grande vs petite agence, etc. Sur les entretiens réalisés, trois ont duré plus de trois heures ; 4) L'analyse des sources doit enfin à une culture d'arrière-plan (formation d'architecte DPLG, expérience acquise dans en agence entre 1984 et 1992, recherches sur la conception architecturale au sein du LAREA). 
«L'importance de la contrainte dans la conception? Mais le travail de conception, ce n'est qu'une somme de contraintes!»

«Le travail de conception, c’est un espace de liberté dans un énorme volume de contraintes... Quand on fait de la conception, on a des contraintes physiques, des contraintes de programme, mais ça reste des points de départ dont on discute et qu'on peut dépasser. Et c'est là qu'il y a un espace de liberté. [...] Gérer les contraintes de la conception, c'est presque de l'ordre du plaisir ; c'est ça qui fait l'intérêt du travail, avec cette logique de l'absurde : plus y a de contraintes, plus on se régale!»

Les deux premiers auto-portraits semblent dépendre d'un effet de «présentation de soi » car, dans les faits, l'architecte n'est jamais ni totalement libre de ses choix, ni totalement contraint par la « cage d'acier » technico-juridique. Des études récentes offrent une piste pour redéfinir cette articulation liberté-contrainte. Boudon et al. écrivent : «La modalité d'exercice de la contrainte en tant que nécessité incontournable est étrangère à l'espace de conception, par définition, puisqu'elle exclut la liberté de choix [...] Mais le traitement de la contrainte se situe à un autre niveau que la contrainte elle-même. La prise en considération d'une contrainte n'exclut pas, elle, des alternatives, donc des choix » (1994 : 157). G'est au fond ce que dit le troisième architecte.

Cette articulation de la liberté et de la contrainte dans le projet peut être clarifiée par un excursus dans la sphère juridique.

1. La loi concède à l'architecte un espace de liberté réel. La loi sur la propriété artistique et intellectuelle du 11 mars $1957^{12}$ définit un droit réel de l'architecte sur ses oeuvres, qui s'étend y compris sur les choix de rénovation d'un bâtiment, dans l'hypothèse où le propriétaire déciderait par exemple de changer la couleur des façades sans l'accord de l'architecte (Cour d’Appel de Paris, 20 novembre 1996). La propriété intellectuelle dont jouit l'architecte, qui repose évidemment sur la contribution originale apportée par le concepteur, est renforcée par le Code des devoirs professionnels ${ }^{13}$ qui prescrit notamment que « le plagiat est interdit » (art.

${ }^{12}$ L'article premier de cette loi : «L'auteur d'une oeuvre de l'esprit jouit sur cette oeuvre, du seul fait de sa création, d'un droit de propriété incorporelle exclusif et opposable à tous » s'applique à l'architecture ainsi que le veut l'article 2 : «Sont considérées comme oeuvres de l'esprit [...] les oeuvres de dessin, de peinture, d'architecture [...] les plans, croquis et ouvrages plastiques relatifs à l'architecture. »

${ }^{13}$ Directement inspiré du code Guadet (1895), resté à l'état de projet par suite de circonstances historiques et politiques défavorables, ce texte fut approuvé par le décret du 24 septembre 1941 avant d'être entériné à la Libération (ordonnance du 16 octobre 1945). 
24 du décret n 80-217 du 20 mars 1980). La notion de plagiat n’a de sens que dans le cadre d'une activité où des productions originales (non typiques) peuvent apparaître.

2. Les rapports de l'architecte avec certains interlocuteurs sont des rapports de contrainte. Par exemple, les rapports entre l'architecte et le représentant de l'État chargé de l'examen des projets ne sont pas exempts de coercition : le permis de construire peut être refusé quand l'architecte transgresse la réglementation en vigueur. Ainsi jugé, dans le cas d'un aménagement dépassant le coefficient d'occupation des sols autorisé. Le permis de construire a été annulé (Conseil d'État, 9 février 1994).

Cet excursus nous assure que les droits artistiques de l'architecte sont réels, de même que les contraintes pesant sur le projet. Mais il nous apprend peu sur la façon dont les concepteurs se saisissent de ces rapports : c'est ce que devrait viser l'étude empirique des pratiques de conception.

\section{Domaine de l'étude}

Ces définitions étant posées, il faut maintenant apporter quelques précisions quant au domaine exact couvert par l'étude des contraintes de conception :

1) Nous n'envisagerons que les mesures coercitives répondant exactement aux définitions précédentes. Nous négligeons la distinction entre avertissement (menace préventive qu'exerce une autorité sur une catégorie de personnes) et tentative de contrainte, en raison de la parenté entre ces notions (Neuberg, 1990). Le Code de la construction, en son titre V : Contrôle et sanctions pénales, art. L 152-1 à L 161-2, le Code de l'urbanisme, en son titre VIII : Infractions, art. L 480-1 à L 480-13, donnent une idée des peines encourues par l'architecte.

2) Nous ne traiterons, dans cet article, que de la contrainte sociale, expression pléonastique pour les sociologues et les philosophes de l'action qui définissent toujours la contrainte comme rapport à autrui, mais non pour les praticiens qui parlent de «contraintes physiques ». Or, lorsque l'homme est confronté à des circonstances extérieures adverses, il n'agit pas par contrainte mais par nécessité (Neuberg, 1990 : 494). Des problèmes de délimitation surgissent d'ailleurs dès que l'on veut suivre l'usage des praticiens ${ }^{14}$. Il existe un critère simple pour

\footnotetext{
${ }^{14}$ Notons que contrainte sociale et contrainte physique se superposent souvent. L'arrêté du 24 décembre 1980, suivant lequel les logements doivent avoir des portes intérieures d'au moins 0,80 m, afin de garantir l'accessibilité aux handicapés, constitue-t-il une contrainte physique ou une contrainte sociale? La contrainte est
} 
savoir s’il y a « contrainte sociale » : c'est, comme le propose déjà Weber (1971 : 68), de lier son existence à un acteur spécifique chargé du contrôle et, le cas échéant, du déclenchement de la sanction. Si un architecte dépose un permis pour un établissement recevant du public dont les plans figurent des équipements électriques non conformes, un acteur spécifique - ici, le représentant de la Commission départementale de la protection civile - émettra un avis défavorable sur le projet (selon le Code de la construction en son art. R 123-35). Il importe peu de savoir si le représentant de l'État se fonde alors sur des données objectives ou non pour émettre son jugement. Sa présence suffit à définir la contrainte ${ }^{15}$. L'existence d'un agent spécifique chargé du contrôle est donc un critère efficace pour circonscrire le domaine des contraintes. Il apparaît que, même sous ces restrictions, les contraintes sont nombreuses et toujours plus nombreuses, pourrait-on dire, en raison de la prolifération législative et réglementaire qui encadre les activités de construction dans la société contemporaine.

3) Enfin, l'étude de la contrainte sera envisagée du point de vue exclusif de celui qui la subit, en l'occurrence le concepteur, et non pas du point de vue de celui qui l'exerce. Les deux optiques sont évidemment défendables, mais, à choisir le second point de vue, il orienterait l'étude, non pas sur la « contrainte », mais sur le « pouvoir » (Chazel, 1992), en particulier sous la forme d'une domination, définie ici par la chance, pour un acteur (maître d'ouvrage, représentant de l'État, contrôleur technique, etc.), de trouver un concepteur prêt à obéir à ses ordres (Weber, $1971: 95)$.

physique parce que la dimension de la porte est calculée à partir des dimensions des fauteuils roulants (largeur, longueur, diamètre de rotation). Mais la contrainte est sociale, parce que l'architecte ne respectant pas l'arrêté s'expose à une sanction, à commencer par un probable refus du permis de construire.

15 En revanche, le géomètre n’a pas de pouvoir coercitif. S'il porte à la connaissance de l'architecte l'existence d'une « contrainte physique » (fort dénivelé, exiguité d'une parcelle, etc.) il ne peut exercer sur lui de sanction : les rapports entre architecte et géomètre sont placés sous le signe de la communication d'informations. 


\section{Le format conditionnel}

La prolifération et l'étonnante disparité ${ }^{16}$ des contraintes s'exerçant sur le projet résultent avant tout de la multiplication des acteurs participant à la conception et à la construction des ouvrages. Un praticien note que « dès le tout début des études de conception, au moins une quarantaine de personnes sont à pied d'oeuvre » (Farel, 1995 : 53). Cet ensemble n'est pas composé que du personnel chargé d'une même affaire au sein de l'agence d'architecture ${ }^{17}$. Il recouvre aussi l'ensemble des intervenants extérieurs auxquels l'agence fait appel durant le projet : maître d'ouvrage (et parfois programmistes et spécialistes du montage d'opération imposés au titre de l'assistance à la maîtrise d'ouvrage) ; services de l'État (services instructeurs du permis de construire, commission de sécurité, architectes des Bâtiments de France, etc.) ; bureaux d'études techniques regroupant divers ingénieurs (ing. structure, ing. fluides, thermiciens, acousticiens, etc.) ; économistes du bâtiment ; entreprises retenues au moment de la passation des marchés et bureaux de contrôle. Chacun de ces acteurs devient, le temps d'un projet, un interlocuteur privilégié de l'architecte, et exerce sur lui une contrainte spécifique.

Au delà des origines diverses des contraintes de conception, il est utile de voir que toutes peuvent être ramenées à un format conditionnel. Les contraintes offrent généralement une forme conditionnelle : si... alors... Par exemple, le Code de la construction stipule en son art. R 111-15 : «L'installation d'un ascenseur desservant chaque étage est obligatoire dans les bâtiments d'habitation collectifs comportant plus de trois étages au-dessus du rez-dechaussée. » On peut aisément traduire cette contrainte sous forme conditionnelle :

${ }^{16}$ «Dans une salle d'opération, un éclairement de 1000 lux est nécessaire »; « Les circuits propre et sale ne doivent pas se croiser dans une salle de restaurant »; «Les cuisines doivent être carrelées jusqu'à une hauteur de 2 mètres »; «Dans un hôpital, les secteurs contagieux et non contagieux doivent être séparés, pour éviter les risques d'infection nosocomiale »; etc.

${ }^{17} \mathrm{Au}$ sein des agences, les activités sont souvent segmentées entre le chef d'agence qui produit l'esquisse, le chef de projet qui veille à son développement et les architectes qui se voient confiés la conception de tel ou tel élément particulier. Ce «modèle hiérarchique » est rarement remplacé par un modèle d'organisation concurrent dit «modèle négocié ». Cette différence d’organisation du travail est perceptible dans la comparaison du fonctionnement des agences de Norman Foster (Londres) et de Renzo Piano (Gênes) (Raynaud, 2001 : 456). 
« Si le bâtiment projeté est un bâtiment d'habitation collectif, et si le bâtiment comporte plus de trois étages au-dessus du rez-de-chaussée, alors l'architecte doit concevoir un ascenseur desservant tous les étages. » (Art. R $111-15)$.

D'autres contraintes adoptent au contraire un format inconditionnel. Ainsi, l'art. R 1237 du Code de la construction prescrit que « Tout établissement doit disposer de deux sorties au moins. » Toutefois, cette proposition est subordonnée à une condition tacite, à savoir que l'article est relatif aux règles de sécurité applicables dans les établissements recevant du public. De plus, le Tribunal administratif de Versailles ayant jugé, le 22 juillet 1998, que ce texte était inapplicable lorsque l'établissement recevait moins de 50 personnes, cette contrainte admet une traduction conditionnelle :

[5] «Si le bâtiment projeté est un établissement recevant du public, et si le bâtiment reçoit plus de 50 personnes, alors l'architecte doit concevoir deux sorties aux moins. » (Art. R 123-8).

Toute contrainte inconditionnelle peut aisément se traduire sous forme conditionnelle ${ }^{18}$. Il suffit d'expliciter la condition, fut-elle omise, sous-entendue, ou si générale que le législateur n’a pas jugé bon de la dire, pour retrouver le format conditionnel. À rebours, une contrainte dont les conditions d'application ne sont pas énonçables ne constitue pas une contrainte légale-rationnelle, car la sanction n'y trouve plus son critère d'application : elle peut frapper n’importe qui, de la façon la plus arbitraire. Il n'est donc pas de contrainte autre que conditionnelle.

\section{Classification des contraintes}

Ce résultat permet d'envisager une classification des contraintes ${ }^{19}$. Partons du format conditionnel. Pour estimer le degré de coercicion du concepteur, il faut considérer le contenu

${ }^{18}$ Le législateur apporterait une aide inappréciable à l'architecte en énonçant les contraintes sous forme conditionnelle. L'architecte accèderait alors plus facilement à l'ensemble des textes réglementaires.

${ }^{19}$ Nous laissons de côté d'autres propriétés intéressantes. Le format conditionnel permet de recenser, à partir du membre si... qui définit la condition d'application, l'origine des contraintes. Un dépouillement des Codes de la construction et de l'urbanisme fait apparaître quelques cinquante contraintes élémentaires réparties en trois registres : contraintes induites par la situation générale du bâtiment (10/50), par son environnement 
de la contrainte dans l'hypothèse où ses conditions d'application sont satisfaites. L'analyse porte sur le membre alors... qui définit l'injonction. Lorsqu'un architecte conçoit un projet, il s'expose, de par la nature spécifique du bâtiment projeté, à des mesures qui peuvent se combiner d'une façon plus ou moins complexe. Nous étudierons tout d'abord les contraintes simples, puis les contraintes composées.

\subsection{Contraintes simples}

Les contraintes simples sont celles qui sont prises isolément — telles qu'elles sont énoncées dans un article de loi, par exemple, et non comme elles se donnent dans un projet réel. Il est possible, en étudiant le contenu des contraintes simples, de distinguer quatre grands types d'injonction :

- L'injonction négative se reconnaît à la forme même de l'énoncé ; c'est une « interdiction ». Rentre dans cette classe l'art. 675 du Code civil, repris par l'art. L 112-9 du Code de la Construction, portant réglementation des servitudes de vue ${ }^{20}:$ «L'un des voisins ne peut, sans le consentement de l'autre, pratiquer dans le mur mitoyen aucune fenêtre ou ouverture en quelque manière que ce soit, même à verre dormant. » (Art. L 112-9). En ce cas, l'architecte devra, soit concevoir des ouvertures sur un mur adjacent au mur mitoyen, soit créer un puits de lumière, soit recourir à un éclairage zénithal, etc.

- L'injonction simplement bornée enjoint le concepteur de respecter, dans les dispositions de l'ouvrage, une borne soit inférieure, soit supérieure, limitant un intervalle donné de liberté. Par exemple, l'art. R 111-17 du Code de l'urbanisme stipule que : «Une distance d'au moins 4 m peut être exigée entre deux bâtiments non contigus. » (Art. R 111-17). En ce cas, l'architecte peut décider librement de la distance entre les deux bâtiments, pourvu toutefois que celle-ci soit supérieure à quatre mètres.

immédiat (29/50), par la nature de l'ouvrage (11/50). Le format conditionnel a aussi des applications en logique déontique que nous rejetons en Appendice.

${ }^{20}$ On trouverait des exemples plus anciens dans les servitudes prédiales urbaines (servitutes praediorum urbanorum) du Droit romain. Citons celle interdisant l'exhaussement d'une construction afin de ne pas nuire aux jours du voisin (jus altius non tollendi), ou celle interdisant de faire tomber sur le fonds voisin l'eau dégouttant d'un toit (jus stillicidii vel fluminis non recipiendi), cf. Accarias (1879). 
- L’injonction doublement bornée a pour particularité d'enjoindre le concepteur de choisir une valeur comprise entre une borne inférieure et une borne supérieure. L’intervalle de liberté est alors doublement borné. Un exemple de ce type d'injonction est fourni par l'art. R 111-19-1 du Code de la Construction, relatif à l'aménagement pour les personnes handicapées des douches et vestiaires des installations sportives : "La zone d'assise, fixe ou mobile, doit avoir une hauteur comprise entre 0,46 m et 0,50 m. » (Art. R 111-19-1). En ce cas, l'architecte se voit contraint de positionner l'assise et la barre d'appui dans les limites étroites définies par la réglementation.

- L'injonction positive est celle qui ordonne à l'architecte de respecter un « ordre » ou une « prescription ", qui n'est soumis à aucune latitude d'interprétation. C'est le cas de l'art. R 123-8 du Code de la Construction portant réglementation des équipements de protection contre les risques d'incendie dans les immeubles recevant du public : "Un éclairage de sécurité doit être prévu dans tous les cas. » (Art. R 123-8). L'architecte doit alors se plier à l'injonction qui lui est faite de prévoir un éclairage de sécurité.

Un apport de la philosophie de l'action doit ici être examiné. Les recherches de Henrik von Wright (1963) montrent que le contenu d'une obligation est plus contraignant que celui d'une permission. L'idée d'assujettir le degré de contrainte au contenu de l'injonction est utile. Est-il possible d'aller plus loin à partir de la classification exposée ci-dessus ?

Prima facie, l'injonction négative semble être très contraignante. Mais, du point de vue du concepteur, elle se limite toujours à une injonction de concevoir qui entrave peu sa liberté de conception. Si l'architecte ne peut percer de vue dans le mur mitoyen, il est certes contraint de concevoir d'autres ouvertures, mais l'art. 675 du Code civil ne lui fixe aucune directive sur la position, le nombre, la forme ou les dimensions qu'il devrait respecter : l'architecte est libre de les concevoir à sa guise. L'injonction simplement bornée exerce une contrainte plus nette sur le concepteur, puisque, en lui demandant par exemple de concevoir un garde-corps de plus d'un mètre, elle se rapproche d'une définition positive du contenu de l'acte. L'architecte reste libre, toutefois, de donner une dimension supérieure à la dimension réglementaire. L'injonction doublement bornée est encore plus contraignante dans la mesure où elle définit positivement le contenu de l'acte de conception, si ce n'est l'intervalle de liberté laissé au concepteur d'adapter le dispositif réglementaire au contexte (une barre d'appui située entre 0,70 m et 
0,80 m, par exemple). L'injonction positive, enfin, est la plus contraignante de toutes, car elle énonce le contenu même de l'acte de conception sans permettre d'interprétation. L'architecte n'a plus aucune prise sur l'action à entreprendre. Un des architectes interrogés a clairement perçu cette différence entre injonction négative et injonction positive :

« Si on me dit: "Moi, je ne veux pas de chauffage central", pour moi, ce n'est pas une contrainte. C'est plutôt celui qui me dit : "Moi, je veux un chauffage électrique". »

En définitive, il existe une relation d'ordre nettement perceptible entre ces injonctions : le degré de contrainte croît de l'injonction négative, qui se contente de proscrire une solution, à l'injonction positive, qui contraint l'architecte à reproduire une disposition préconçue et étrangère à son choix. Ce résultat quelque peu paradoxal est aisément compréhensible quand on le rapporte aux activités de conception. Ce qui est contraignant pour le concepteur, c'est de traduire en espace le contenu de l'injonction, et d'ajuster ce contenu aux formes et aux dimensions du bâtiment. Plus le contenu de l'injonction se rapproche d'une définition positive de l'espace construit, plus il empiète sur les choix de l'architecte. Au contraire, l'injonction négative ne pas s'immisce pas dans le travail de conception : une interdiction signifie seulement que, parmi toutes les hypothèses que l'architecte est libre d'évoquer, une ne doit pas être retenue.

Mais il est également possible que ce paradoxe ne soit au fond que la conséquence d'une représentation fondamentalement erronée des rapports entre liberté et contrainte, à la fois dans l'ordre de la poïésis et de la praxis - pour reprendre une distinction aristotélicienne. En ce cas, le problème ne serait en rien spécifique à l'architecture. C'est ce à quoi nous convie la philosophie analytique de l'action et notamment la définition [1]. Son succès tient au fait qu'elle résout adéquatement le paradoxe signalé. Pourquoi interdire est-il moins contraignant que prescrire? Une prescription impose une action prédéterminée ; une interdiction autorise au contraire une liberté dans le choix de l'action différant de l'action interdite («Je peux donc choisir d'agir autrement »). La définition classique, ou toute révision qui conserverait l'idée d'une définition négative de la liberté, est incapable d'expliquer ce fait. 


\subsection{Contraintes composées}

Les contraintes composées résultent de la composition des contraintes simples en une même partie de l'ouvrage. La composition des contraintes est susceptible de faire changer la nature de la contrainte résultante, au vu de la classification précédente. Bien qu'il n’existe pas de règle générale de composition, on constate que la composition équivaut : 1) soit à réitérer l'injonction la plus restrictive des deux contraintes initiales ; 2) soit à formuler une injonction résultante plus contraignante que l'une des deux contraintes initiales prise isolément (l'injonction résultante ne peut jamais être moins contraignante que les contraintes de départ). Examinons ces deux cas.

Considérons tout d'abord le cas d'un architecte chargé, par un maître d'ouvrage public, de concevoir un équipement sportif. Supposons que ce maître d'ouvrage ait un budget limité à sa disposition. En veillant à la dépense, il exercera une contrainte économique sur le concepteur. Par ailleurs, le bâtiment étant classé établissement recevant du public, il doit être accessible aux personnes handicapées, conformément aux arts. R 111-19 à R 111-19-3 du Code de la Construction. Ces deux contraintes se combinent sans modifier la marge de liberté de l'architecte, quant à la conception des douches et des vestiaires pour handicapées :

« Si Le montant global des travaux est minimum. » (Maîtrise d'ouvrage).

«Si La zone d'assise, fixe ou mobile, des douches et des cabines pour handicapés doit avoir une hauteur comprise entre 0,46 m et 0,50 m. » (Contrôle technique selon l'art. R 111-19-1).

«Alors l'architecte doit dessiner une zone d'assise des douches et des cabines pour handicapés à une hauteur comprise entre $0,46 \mathrm{~m}$ et $0,50 \mathrm{~m}$. »

L’injonction réglementaire est insensible à la contrainte exercée par le maître d'ouvrage, car le choix d'une hauteur d'assise n'a pas d'incidence sur le montant des travaux. L'injonction résultante reproduit alors l'injonction la plus contraignante des deux contraintes initiales.

Considérons ensuite l'exemple d'un architecte travaillant, pour le compte d'un promoteur, sur un projet mixte de bureaux et logements en IGH (immeuble de grande hauteur, tel que le plancher bas du dernier niveau soit à une altitude $\mathrm{h}>28 \mathrm{~m}$ ). La 
consultation de la Commission départementale de la protection civile étant obligatoire, on peut imaginer que le concepteur ait à gérer des contraintes contradictoires de la part du promoteur et du représentant de la Commission de sécurité :

«Si la surface habitable doit être maximum. » (Maîtrise d'ouvrage).

«Si le montant global des travaux doit être minimum. » (Maîtrise d'ouvrage).

«Si l'ascenseur doit avoir une capacité minimum de 8 personnes, le nombre et la largeur des sorties et des dégagements intérieurs devant être proportionnés au nombre de personnes appelées à les utiliser. » (Commission de sécurité, selon art. R 123-7).

«Alors l'architecte doit dessiner un ascenseur de 8 personnes seulement. »

Les contraintes exercées par le maître d'ouvrage et le représentant de la Commission de sécurité sont deux injonctions simplement bornées, mais de sens opposé. Si l'architecte concevait un ascenseur de 6 personnes, il dérogerait aux ordres de la Commission de sécurité. Si l'architecte dessinait un ascenseur de 10 personnes, il dérogerait aux injonctions du maître d'ouvrage. En affectant une part de la surface hors oeuvre brute à l'ascenseur, il réduirait d'autant la surface habitable des appartements et des bureaux. En choisissant un ascenseur plus cher, une part du budget serait affectée aux communs, au détriment des appartements et des bureaux. La composition de deux injonctions simplement bornées, mais de sens opposé, équivaut donc à une injonction positive. C'est pourquoi l'architecte n'a d'autre choix que de dessiner un ascenseur de huit personnes.

\subsection{Contraintes de forme et de disposition}

Même dans le cas où le travail de l'architecte consiste à reproduire des contraintes prédéfinies a minima, il existe des raisons de croire que la « cage d'acier technico-juridique » ne s'est pas totalement refermée sur lui. En effet, il faut distinguer les injonctions relatives au principe et celles relatives à la disposition de l'ouvrage. Considérons les deux cas suivants :

« Les dimensions d'une salle de handball homologuée sont : longueur $40 \mathrm{~m}$, largeur $20 \mathrm{~m}$, hauteur $7 \mathrm{~m}$, zone supplémentaire sans obstacle sur les côtés latéraux $1 \mathrm{~m}$, sur les côtés frontaux $2 \mathrm{~m}$. » (Fédération internationale de handball). 
«Dans un établissement recevant du public, un éclairage de sécurité doit être prévu dans tous les cas. » (Commission de sécurité selon Art. R 123-8).

La première injonction fixe l'implantation et la forme de l'ouvrage. En ce cas, l'architecte reproduit un modèle sans faire de réel travail de conception. C'est une injonction de disposition. Si des contraintes économiques émanent du maître d'ouvrage, il est facile de prédire que la salle de sport aura la forme d'un parallélépipède de 44 m x 22 m x 7 m, auquel sera adossé un bloc de services (douches, vestiaires, local de l'entraîneur, etc.) aux formes à peine plus libres. C'est un cas rare. Ainsi, la seconde injonction exige de l'architecte de prévoir un dispositif de sécurité, sans le contraindre dans ses choix d'implantation, de forme ou d'aspect. Il pourra donc concevoir librement un tel dispositif, le texte ne fixant pas la forme de l'ouvrage, mais seulement le principe auquel l'architecte doit répondre. C'est une injonction de principe. Sauf cas rare, l'architecte affronte des injonctions de principe qui se soumettent entièrement au critère d'incomplétude et qui, de ce fait, plaident pour la révision forte de la définition de la liberté opérée par la philosophie de l'action. Sinon, comment l'architecte pourrait-il être libre en respectant la contrainte?

\subsection{Contraintes latérales}

À la fin de son étude sur la contrainte, Nozick (1991) identifie des clauses permettant de reconnaître l'existence de « cas non centraux de contrainte » qui entretiennent certaines relations avec les « contraintes centrales » répondant aux critères [2-3] du $\S 1.2$. Il est utile de déterminer si des contraintes autres que les injonctions positives peuvent rentrer dans cette catégorie. Si tel était le cas, en effet, la situation de l'architecte pourrait s'avérer plus coercitive qu'il n’a été dit. Deux des clauses énoncées par Nozick (1991) sont applicables aux activités de conception. Nous les discuterons tour à tour.

«Si $\mathrm{P}$ contraint $\mathrm{Q}$ à faire $\mathrm{A}$ et si la seule façon de faire $\mathrm{A}$ est de faire $\mathrm{B}_{1}$, ou $\mathrm{B}_{2} \ldots$ ou $\mathrm{B}_{\mathrm{n}}$, alors $\mathrm{P}$ contraint $\mathrm{Q}$ à accomplir $\mathrm{B}_{1}$, ou $\mathrm{B}_{2} \ldots$ ou $\mathrm{B}_{\mathrm{n}}$. » (Nozick, 1991 : 279).

Cette clause est en général inapplicable en architecture, car, comme l’a noté Herbert A. Simon (1969 : 84), le concepteur ne peut atteindre que des solutions sous-optimales, valant 
satisfecit. Ces solutions ne forment donc pas un ensemble prédéfini et dénombrable. Les solutions étant imaginées par l'architecte, la transmission de la contrainte est rompue, sauf à supposer que l'interlocuteur de l'architecte énonce lui-même les termes d'un choix obligatoire entre plusieurs injonctions positives. Dans tous les autres cas, l'architecte est libre d'imaginer la solution qui lui convient, pour autant qu'elle ne contrevienne pas à la réglementation. Par exemple, si un représentant de la Commission de sécurité contraint le concepteur à modifier sa distribution pour veiller à l'évacuation du public, il ne le condamne pas à adopter l'une des solutions $\mathrm{B}_{1}$, ou $\mathrm{B}_{2} \ldots$ ou $\mathrm{B}_{\mathrm{n}}$, car c'est l'architecte qui définit le nombre et la nature des solutions qu'il lui plaît de concevoir.

« Si $\mathrm{P}$ contraint Q à faire $\mathrm{B}_{1}$, ou $\mathrm{B}_{2} \ldots$ ou $\mathrm{B}_{\mathrm{n}}$, et si l'action $\mathrm{B}_{1}$ est la meilleure de la série des $\mathrm{B}_{\mathrm{i}}$ (la seule qu'il est raisonnable de faire, etc.), et si $\mathrm{Q}$ a fait $\mathrm{B}_{1}$ pour cette raison, alors $\mathrm{P}$ a contraint $\mathrm{Q}$ à accomplir $\mathrm{B}_{1}$. » (Nozick, $1991: 279)$.

Gette autre clause est également inapplicable à la conception architecturale, même si l'action de l'architecte est rationnelle (justifiable). Car son action ne se soumet à cette clause que si les solutions peuvent être classées selon leur pertinence, relativement au problème posé. C'est précisément ce classement des propositions architecturales qui fait défaut. Les solutions satisfecit de Herbert A. Simon n'apparaissent que dans des contextes où la maximisation et l'optimisation n'ont pas cours. Cet obstacle rend alors la clause précédente inapplicable à la conception. Lorsqu'un architecte des Bâtiments de France contraint le concepteur à modifier sa façade, en raison de la proximité d'un monument classé, il ne le contraint pas à adopter la solution " la plus raisonnable », car seul l'architecte est en mesure de définir quelle est la solution raisonnable, et en fonction de quoi elle lui paraît telle.

Il ressort de cet examen des contraintes latérales énoncées par Nozick, que les clauses auxquelles on reconnaît l'extension ou le prolongement d'une contrainte ne sont opérantes que sur les injonctions positives. Dans tous les autres cas, la contrainte ne s'étend pas à la nature même de la transformation à entreprendre, et la seule contrainte qui s'exerce sur l'architecte est alors une exigence de conception dégageant un nouvel espace de liberté. 


\section{Stratégies du concepteur}

Au-delà de l'énoncé général selon lequel l'acteur jouit toujours d'une marge de liberté dans une situation de contrainte, Crozier et Friedberg s'engagent parfois dans une description beaucoup plus fine de la façon dont les acteurs se saisissent des contraintes. Ils écrivent:

« Telle règle ou telle prescription formelle qui apparaissent tout d'abord comme des contraintes seront "détournées" de leur sens. [...] Le meilleur exemple [montrant que les règles ne suppriment pas toutes les sources d'incertitude] est fourni par les négociations et marchandages qui ont lieu autour de l'application de la règle. [...] La structure formelle ne règle pas tout, les acteurs disposent toujours d'une marge de manœuvre qu'ils utilisent pour interpréter, manipuler et biaiser les prescriptions qu'elle contient » (1977 : 43, 88, 147, mes italiques).

Détournement, interprétation, manipulation, etc. sont autant de stratégies que les acteurs peuvent utiliser pour se préserver une marge d'action. Il n'en va pas différemment dans le travail de conception. Évidemment, l'architecte doit parfois se soumettre entièrement à la contrainte et respecter le texte à la lettre, ainsi que le dit un architecte ${ }^{21}$ :

«Avec le service incendie, c'est bloqué. Il ne faut pas que le rebord de la fenêtre soit plus haut que $8 \mathrm{~m}$; à 8,02, ils refusent. Si la borne incendie est à $52 \mathrm{~m}$ au lieu de 50 : ils refusent. Ce manque de souplesse, c'est aberrant! Les pompiers, c'est pas Montesquieu. C'est la loi, toute la loi, mais c'est pas L'Esprit des lois !»

Toutefois ce constat suggère en filigrane deux ouvertures stratégiques : la négociation avec un supérieur hiérarchique ou l'interprétation du texte - cas qui se présente y compris dans la discussion avec les services de sécurité incendie.

${ }^{21}$ L'Institut du Monde arabe (concours gagné par Jean Nouvel en 1981) en fournit un exemple. L'esquisse présentait initialement une côte d'altitude de $38 \mathrm{~m}$, dérogeant ainsi au gabarit urbain imposé en front de Seine en rentrant dans le champ de visibilité du Panthéon. Les concepteurs ont dû modifier leur projet : ils ont réduit la hauteur du bâtiment de 6 mètres. La hauteur sous plafond est ainsi passée de 2,70 m à 2,10 m dans les étages courants. Mais, comme le remarque l'un des architectes interrogés, le sentiment de contrainte ne résulte-t-il pas ici de ce que les concepteurs ont intégré trop tard ce gabarit à leur réflexion? D’autres solutions étaient également envisageables (diminuer la surface de plancher, localiser des collections en sous-sol, etc.) Les architectes ont donc pris la décision de diminuer la hauteur sous plafond contre d'autres éventualités, parce que ce choix leur permettait de limiter l'incidence et le coût des remaniements à l'étude du passage des gaines dans la poutraison. 
Les témoignages recueillis font ressortir la diversité des stratégies que les concepteurs adoptent face aux contraintes qui leur sont imposées. Au delà des contextes, on peut identifier quelques grands types de réaction à la contrainte qui répondent au cadre de l'analyse stratégique cum grano salis - en raison de la composante axiologique éludée par ce programme 22. Commençons par un tour d'horizon, que nous ne jugeons pas exhaustif.

\subsection{Négociation de la contrainte}

Si les acteurs chargés du contrôle appliquent souvent les textes sans irénisme, ils ont à portée de main tout un arsenal de dérogations prévues par le droit. Plus fondamentalement, Erhard Friedberg a montré qu'une règle ne consiste pas à « déterminer directement des comportements, mais à structurer des espaces de négociation et de jeu entre les acteurs » (1992 : 536). Ce sont là deux raisons justifiant le recours à la négociation (Thuderoz, 2000). L'une des stratégies consistera à réduire la contrainte en jouant directement sur l'agent en charge de son application :

« [Concernant les services instructeurs] il y a l'institution et puis, à l'intérieur, les hommes. Si tu tombes sur un tatillon qui ne veut pas en démordre, la négociation sera évidemment difficile. »

« Je ne remets pas en cause la fonction de l'ABF, qui est utile, mais enfin il y a des choses plus importantes que de savoir s'il faut mettre telle pierre. Toujours est-il qu'il faut savoir négocier ces contraintes. Comme c'est surtout une affaire d'individus, j'essaie de savoir à qui j'ai affaire. Il faut cerner ce qui va les titiller et y répondre.

${ }^{22}$ La composante axiologique est bien attestée. Interrogé sur les mentions du Cahier des clauses administratives particulières qui définissent des possibilités de contraintes relativement au contenu des prix, un architecte dira, visiblement embarrassé : «Alors ça, c’est zéro. C'est vraiment une parade qu'on ne peut pas utiliser. J'aime pas... ce serait vraiment la dernière extrémité, si j’étais en procès et si j’étais obligé de me défendre. Je trouve ça... [soupirs]. Il faut pas se servir de ça!» Tous les coups ne sont donc pas permis et l'architecte juge aussi de l'action entreprise « en son âme et conscience », en fonction de principes normatifs sous-jacents qui pondèrent son oportunisme (Boudon, 1992 : 39). Friedberg (1993 : 210-221), tout en reconnaissant les limitations culturelles et normatives de l'action, maintient que la différence webérienne entre Zweckrationalität et Wertrationalität est une clarification ex post, qui n'intervient pas ex ante, au moment où l'acteur fait son choix. Sur cette question, le désaccord persiste entre le paradigme stratégique et le paradigme actionniste (cf. Boudon, $2003: 48)$. 
Il faut aussi leur laisser quelque chose : il faut qu'ils aient quelque chose à dire, sinon ils ne justifient plus leur rôle ; ça aussi c'est une tactique. »

« Pour en revenir aux pompiers, dans cette partie du bâtiment, il y avait un escalier à vis malcommode et dans un état déplorable. Le problème était : ou on le change et on est obligé d'être réglementaire : giron de 28, hauteur de 17, etc. ; ou on n'y touche pas, sinon je suis responsable. Les pompiers ont dit : "Mais nous, on ne peut pas accepter !' C'était à nouveau la quadrature du cercle. Finalement, c'est le service d'urbanisme de la ville qui à fait céder les pompiers. Il faut savoir que certains services ont le pouvoir sur d'autres. »

« Il y a aussi l'éternelle querelle entre les architectes et les ingénieurs. On a tendance à reprocher à l'ingénieur de ne pas avoir beaucoup d'imagination, de ne pas chercher des solutions intéressantes, et nous on est là pour leur dire... C'est évident que ça fait partie du jeu ! Avec les bureaux d'études structure, fluides, etc. c'est important de choisir des gens qui vont essayer de trouver les solutions les plus astucieuses. On aime bien travailler avec les “je-trouve-tout” ! Et à ce jeu, c'est souvent les ingénieurs des Arts et Métiers qui sont les meilleurs ; ils ont ça dans l'âme. »

«Avec l'architecte des Bâtiments de France il y a énormément de négociation. Ils ont un droit de regard effrayant et le problème c'est qu'ils n’ont rien d'écrit, au contraire des pompiers ! Donc, tout se passe en négociation, avec ce travers que, selon la personne qu'ils ont en face, ils n’ont pas la même réponse. »

« Il y a aussi une part croissante de négociation avec les bureaux de contrôle. À cause de la logique de contentieux généralisée, ils ont tendance à ouvrir le parapluie plus grand que nécessaire. Il y a donc une marge de négociation. »

Ces négociations sont parfois mal perçues par les concepteurs : elles distendent le temps du projet, fait sur lequel nous reviendrons en conclusion.

Nous avons commencé cette typologie par la négociation, qui fait aujourd'hui l'objet de toutes les attentions en sociologie du travail. Cette focalisation ne doit cependant pas faire oublier que la négociation n'est qu'une des stratégies imaginables pour conquérir une marge d'autonomie.

\subsection{Interprétation de la contrainte}

Une deuxième stratégie d'autonomisation s'appuie sur le fait que toute contrainte est soumise à interprétation. C'est ce que reconnait implicitement un contrôleur de la Socotec :

«Moi, je suis impressionné par le fait que les architectes sont drôlement forts pour respecter les normes en inventant à chaque fois des solutions nouvelles. C'est là qu'on voit le métier. » 
Mais d'où vient cette liberté d'interprétation? L'analyse de quelques cas révèle que la condition d'application de la contrainte ne peut avoir de réelle portée que si elle est générale ; un énoncé général décrit imparfaitement une situation concrète ; la règle doit être ajustée à la situation et cet écart est une source de liberté. L'interprétation naît de l'incomplétude de la contrainte. C'est ce que restituent certains entretiens :

«Avec les pompiers, il y a des tels enjeux [de sécurité des personnes] que ce n’est pas vraiment de la négociation. C'est une forme de dialogue : "Si on peut pas faire X est-ce que, si on fait Y, ça marche ?" Et le gars dit oui ! Il y a toujours une marge d'interprétation, mais c'est pas de la négociation. [Pour les services incendie] tout se joue sur "sortir". Il y a un moment où ils finissent par dire : "Là on est d'accord. Vous sortez sur le toit, donc on considère que vous êtes dehors ; on pourra toujours venir vous chercher." Évidemment, ça dépend beaucoup de la personne qui est en face, mais en général c'est clair. C'est vraiment une négociation a minima. Je n'ai jamais vu un bâtiment déclassé par les pompiers de la $3^{\mathrm{e}}$ à la $2^{\mathrm{e}}$ catégorie pour les beaux yeux d'un architecte $[\ldots]$ Les catégories, elles sont définies objectivement. »

«En amont, il y a aussi le géotechnicien qui va faire les sondages. Ça aussi, ce n’est pas parole d'évangile. Loin de là ! - Il ne fait pas une traduction fidèle de la réalité ? - Non, il y a une grande part d'interprétation. On a souvent de longues discussions pour qu'il justifie le positionnement de pieux à telle distance, etc. Après, il faut lui faire entériner ce à quoi on veut arriver. C'est différent du géomètre, à qui on demande seulement de retranscrire la topographie avec exactitude. On n'a pas à négocier avec lui. Le géotechnicien, il dit : "Vu le terrain, la grave à 2 m 50, etc., il vous faut absolument un soubassement. - Mais pourquoi ?" Comme c'est l'architecte qui gère les contraintes économiques, on leur dit : "Il faut faire un effort !" Notre compétence est souvent prise de court parce qu'on n'a pas les mêmes qualifications, mais on peut discuter sur l'interprétation des résultats ; on a les connaissances pour ça. »

\subsection{Détournement de la contrainte}

Le cas du détournement apparaît dans le témoignage d'un architecte interrogé sur les savoir-faire circulant dans les agences d'architecture :

«Pourrait-on parler de la tactique, bien connue en agence, qui consiste à lier deux bâtiments indépendants par une arche pour assurer la continuité du bâti ? - C'est une question de prospect [...] Dans tous les PLU, il y a des règlements de distance minimum à respecter entre deux bâtiments qui sont sur le même terrain. À partir du 
moment où ils sont liés par un petit bout d'arche, c'est le même bâtiment, donc il n'y a plus à tenir compte du prospect réglementaire.»

Le détournement consiste à introduire une modification (mineure) dans le projet de manière à suspendre l'application de la contrainte. Dans le cas cité : les règles de prospect sont applicables à des bâtiments différents ; si deux bâtiments sont liés, ils ne forment qu'un ; donc la contrainte n'a plus de point d'application. Mais tout aussi intéressante est la suite de l'entretien sur la diffusion de ces savoir-faire :

« D’ailleurs, il commence à y avoir une contre-réaction! Certaines mairies ont compris le truc. Les maires, qui trouvent le bâti trop dense, commencent à dire : "De ça, on n’en veut pas. Vous respectez la règle !" - Ça veut dire que ce savoir-faire est connu à l'extérieur? - Oui, maintenant c'est sorti des agences. »

\subsection{Rejet argumenté de la contrainte}

Il existe d'autres stratégies face à la contrainte. L'une d'elle a été popularisée par le concours international de la Bibliothèque d'Alexandrie, lancé par l'Unesco et la République égyptienne en 1989. Le cahier des charges du programmiste stipulait que le bâtiment devait être sans sous-sol, parce que, étant situé à une dizaine de mètres de la mer, il risquait d'être inondé et soumis aux effets de la corrosion saline. L'équipe norvégienne de Snøhetta a tout simplement ignoré cette règle. Les concepteurs ont accompagné leur projet d'une lettre au jury faisant valoir que le programmiste était sorti de sa mission. Son travail était de décrire le problème (proximité de la mer, salinité, corrosion), pas de proposer une solution technique, tache qui incombe exclusivement au concepteur (on sait par exemple construire un sous-sol à l'aide d'un cuvelage). Le jury a reconnu le bien fondé de l'argument et a, compte tenu des autres critères, classé premier le projet de Snøhetta.

Ce cas est évidemment spectaculaire, mais la vie quotidienne des agences regorge de stratégies identiques appliquées à des problèmes de moindre ampleur :

« La contrainte, c'est vrai qu'on est obligé de s'y frotter. Dans ce projet, j’ai essayé de faire passer deux ou trois idées. Alors il faut convaincre les gens qu'il y a des contraintes un peu absurdes. [...] Dans ce projet, il y avait notamment des contraintes liées à une copropriété voisine qui disait : "Rendez vous compte ! Vous allez nous faire de l'ombre." Alors, il faut convaincre les gens. Si on fait une projection sur la maquette, on s'aperçoit 
que l'ombre portée ne tombe pas à l'endroit qu'ils pensaient ! [...] À un moment donné, tu mets aussi les gens devant leurs contradictions... Ça peut marcher. Mais quelquefois on sait qu'on va au combat. Il faut évaluer le rapport de force et toujours garder à l'esprit que certaines contraintes sont cependant justifiées. »

« [Dans telle ville], on peut faire des bow-windows. Pour moi, un bow-window c'est un balcon fermé en projection sur la rue. Après, c'est à l'architecte d'interpréter où il met ses fenêtres... s'il le fait courbe ou carré... Et bien non ! Pour Mme X, Chef du Service d'urbanisme, un bow-window, c'est un balcon qui a deux fenêtres sur les côtés. Là, c'est de l'abus de pouvoir ! [...] C'est passé au-dessus, par la voie hiérarchique, jusqu'à ce que l'élu dise : "Ce n'est pas contradictoire." C'était uniquement une question de responsabilité. Bon, je comprends aussi les contraintes des services instructeurs, mais en rentrant dans une logique de plus en plus procédurière, ils interprètent dans un sens trop strict. Tu dois te déplacer, expliquer et argumenter. Et si tu n'argumentes pas, ça ne passe pas. Si j’avais rencontré ce cas, j’aurais pris une journée entière pour éplucher la bibliographie, pour photographier quelques bâtiments et pour lui montrer qu'un bow-window c'est pas ce qu'elle croit. »

Signe (improbable) de vitalité de la profession : les arguments de parade se diffusent à la même échelle que la contrainte à laquelle ils répondent. Si la contrainte est spécifique à un projet, l'architecte est seul à pouvoir en faire la critique ; si la contrainte se diffuse à une échelle plus large, il y a toutes les chances que les revues professionnelles se saisissent du problème. C'est le cas du rapport SHONSU recommandé par les programmistes aux maîtres d'ouvrage pour juger de la rentabilité des projets : sa critique est désormais publique ${ }^{23}$.

\subsection{Absorption de la contrainte par anticipation}

Certains architectes que nous avons interrogés font également apparaître un autre type de réaction. En situation de projet, le concepteur nourrit régulièrement des expectations sur le

${ }^{23}$ Le rapport SHONSU est le rapport entre la surface hors œuvre nette (SHON) et la surface utile (SU). Plus ce rapport est proche de 1, plus le projet est jugé rentable. « Les programmistes expliquent de façon très persuasive aux maîtres d'ouvrage que l'on sait maintenant construire des universités avec un SHONSU de 1,22 et lorsque l'architecte patine péniblement à 1,5 c'est qu'il ne sait pas s'y prendre » (Michelin, 2003 : 30). L'auteur objecte le flou de la définition de la SU : les halls et les sanitaires ne sont pas toujours comptabilisés, ce qui modifie considérablement la base de calcul. Il montre que les architectes français ont réagi plus lentement que leurs confrères européens à ce critère de rentabilité. Il examine ensuite une à une les possibilités d'action du concepteur. Conclusion : les praticiens doivent tranformer cette pratique en éduquant les maîtres d'ouvrage à juger leurs projets sur la base du rapport SHOBSDO entre la surface hors œuvre brute (SHOB) et la surface dans l'œuvre (SDO), rapport jugé « beaucoup plus pertinent » par l'auteur de cette critique. 
comportement de ses interlocuteurs ; il peut de cette manière parer certaines contraintes en anticipant leur application. Une ligne de conduite consiste à absorber, par des marges dimensionnelles ou financières, d'éventuelles modifications ultérieures :

«Avec le cadastre, une ou deux fois, je me suis fait piéger, parce que le cadastre était faux. Tu te retrouves dans un cas de figure où, au lieu d'avoir un prospect de $5 \mathrm{~m}$, il est réduit à $3 \mathrm{~m} 50$ ! Alors qu'est-ce que tu fais ? »

«Quand j'attaque les choses au stade de l'esquisse [...] je sais que je me garde des marges. La conception, c'est quelque chose de flou qui se précise au fur et à mesure. Ça peut être au niveau du dessin. C'est systématique : je sais qu'on montera de la cloison de 5 ou de 7, mais je dessine 10 [...] Ces centimètres, je les garde en réserve. Je sais qu'ils me seront utiles à l'exécution. Il manque toujours quelques centimètres, parce qu'on n'a pas fait un relevé précis du terrain ou parce que le maître d'ouvrage ne voulait pas engager des dépenses à ce moment-là. [La stratégie,] c'est de garder des marges dimensionnelles ${ }^{24}$. C'est presque une démarche générale ; même au niveau financier. J'ai tendance à tirer les prix vers le haut. Comme ça, je sais qu'on pourra revenir en arrière quand on aura les métrés. Donc je garde des "poches” qui permettent de rattraper des oublis. Ensuite, si c'est trop cher, on peut enlever des bricoles; sinon tu te retrouves à déshabiller complètement le projet [...] Cette logique de marges, j'essaye de la conserver le plus longtemps possible [...] C'est ça, mon approche, et je n'en parle pas trop. Globalement, ça permet, face au maître d'ouvrage, d'avoir une logique de responsabilité et de sérieux. »

«Je me couvre, quand même, quand ça dégénère. J'envoie des lettres recommandées. Il faut se border sans arrêt. Il faut savoir que ça peut aboutir à un procès à la fin d'un chantier. Il y a, dans le métier, cette exigence de faire les choses en règle... et de ne pas donner des verges pour se faire fouetter ! Il faut vraiment se border et làdessus, je trouve que la formation des architectes est très insuffisante. »

On notera que le deuxième architecte lie sa capacité à absorber les contraintes à l'image qu'il donne de soi au maître d'ouvrage. Cela rappelle que la gestion des contraintes est un élément-clef de l'expertise professionnelle de l'architecte ${ }^{25}$.

${ }^{24}$ Cet architecte mentionnera à la suite plusieurs contre-exemples : un projet où les gaines avaient été mal dimensionnées ; un projet dans lequel des joints de dilatation avaient été oubliés alors que le bâtiment était calé sur les prospects et que les surfaces étaient contractuelles!

${ }^{25}$ Un autre architecte explique en ces termes les difficultés inhérentes à la stratégie des marges : « [Cette tactique] suppose une grosse habitude professionnelle. Il faut déjà avoir un peu d'entraînement. On ne peut pas non plus raconter trop d'histoires au maître d'ouvrage, sinon ça peut devenir trop visible. Dans le descriptif, il faut prévoir des quantités un peu supérieures, mais après il faut négocier avec l'entreprise parce que, quand les marchés sont signés, l'entreprise peut dire : "Ah oui, mais c'est au marché, on revient pas dessus...” ". Pour une étude des compétences professionnelles de l'architecte, cf. Raynaud (2001). 


\subsection{Recours à la contre-contrainte}

Une autre stratégie utilisant toutes les ressources de l'anticipation consiste à contraindre celui-là même qui pourrait ultérieurement exercer une contrainte sur l'architecte :

«On peut se servir d'une contrainte pour contraindre l'autre! Il faut résister à cette tendance qui consiste à nous demander toujours plus de modifications. Moi, j'essaye de mettre une borne, parce qu'il n'y aucune raison de s'arrêter ! Le temps est un levier : le permis de construire est valable deux ans ; l'enveloppe budgétaire c'est sur l'année parce la dotation doit être inscrite pour subvention au Conseil général ; une école doit ouvrir en septembre, etc. Ça nous permet de dire : "Maintenant, on arrête !" [Dans un autre projet], il y avait des contraintes de prospect et puis, par ailleurs, une demande urgente de relogement des gens du quartier qui allaient se retrouver à la rue. "Alors si voulez qu'on fasse ça rapidement, il faut accepter de passer outre ce règlement qui est aberrant”. Je ne peux pas dire précisément à quel endroit j’ai joué, mais ça a marché. »

«Les programmes sont rarement bien faits. Ils sont souvent lapidaires, incohérents. Donc, moi, je travaille sur le programme. Ça fait partie d'une démarche qui consiste à bien cadrer les besoins du maître d'ouvrage, à les exprimer d'une façon claire. Sinon, on prend le risque de les découvrir après coup. Et ça, c'est dur : tu pars sur une idée et puis tu t'aperçois que c'est pas comme ça qu'il voyait les choses. Ça fait partie de ma méthode : travailler beaucoup avant de dessiner quoi que ce soit. Il y a une autre conséquence à ça : on peut dire au maître d'ouvrage qu'on a répondu à sa programmation et que, à partir du moment où il demande autre chose, il doit l'assumer [financièrement]. »

Rentre également dans cette catégorie l'observation selon laquelle, plus un ouvrage est dessiné et décrit, moins il est modifiable, et plus le concepteur est maître de ses choix :

« Ce qui n’est pas dessiné n’est pas conçu. C’en est presque un axiome! Les problèmes qui ne sont pas réglés au moment de la conception en disant on verra plus tard, c'est ça qui posera le plus de problèmes sur le chantier. Pour ce qui est de mes affaires, je dessine le bâtiment complètement ; ou alors il y a un endroit que je ne dessine pas, mais qui ne me bloquera pas parce que j’ai déjà anticipé toutes les variantes. Cet endroit, je ne l'étudie pas si je sais qu'il ne va pas tout remettre en cause. »

Il est intéressant de voir que les plans sont eux-mêmes conçus comme un lieu d'exercice de la contre-contrainte, s'agissant de documents contractuels. Un des architectes observe que dans le CGAG et le CGAP (Cahier des clauses administratives générales/particulières), il existe toute une batterie de mentions légales laissant toute ambiguité résiduelle à la charge de 
l'entreprise. Afin d'éviter toute contradiction entre les documents, il existe un ordre défini de préséance entre les pièces écrites et les pièces graphiques. Quant aux pièces graphiques, « le plan à plus grande échelle prévaut en cas de contradiction. » Et si un point du descriptif est inexact, si deux documents ne se correspondent pas exactement, les entreprises seront tenues de réaliser l'ouvrage conformément aux plans d'exécution qu’ils ont signés.

\subsection{Phagocytage de la contrainte}

Il existe aussi des formes plus radicales d'absorption de la contrainte, dont l'une a été utilisée par Kazuo Shinohara dans un projet de maison individuelle. Face à une parcelle exiguë et traversée par une ligne à haute tension, l'architecte a complètement réinvesti la contrainte en percevant le gabarit de sécurité comme une source d'expressivité :

«Pour ne pas réduire le volume de l'étage, Kazuo Shinohara décida de suivre le gabarit déterminé par la réglementation protégeant l'ensoleillement des maisons voisines, d'où le biais de la façade nord, et par les deux espaces cylindriques définis autour des lignes électriques [...]»(Goulet, $1983: 41$ ).

Dans le contexte réglementaire français, un des architectes dit avoir traité de la même manière le gabarit d'une conduite. Un autre nous a signalé le projet d'Éric Gouesnard pour une maison près de Sarzeau, dans lequel le règlement d'urbanisme obligeait à une couverture en ardoise. Le maître d'ouvrage ne voulant pas d'une architecture régionale, l'architecte lui a proposé deux "monolithes" entièrement recouverts de dalles d'ardoise (Gouesnard, 1999 : 6263). Un autre dit avoir renversé la contrainte d'une falaise instable — situation dans laquelle les règlements obligent à un retrait du bâtiment égal à la moitié de la hauteur de la falaise en concevant un «bâtiment-mur de soutènement ».

Dans chacun de ces cas, on assiste à une transgression originale de la contrainte qui consiste, non pas à n'en pas tenir compte, mais à trouver, tantôt dans le respect à la lettre de la contrainte, tantôt dans son application par excès, une chance d'être aux antipodes du principe qu'elle énonce (la règle sur les lignes électriques n'est pas destinée à faire épouser le gabarit de sécurité ; le règlement sur les toits en ardoise ne vise pas à promouvoir l'architecture contemporaine ; etc.) La liberté d'action est ici conquise en détournant non pas la lettre, mais l'esprit de la loi. Ces cas de phagocytage ont une portée théorique : ils répondent à la définition [1] de Chisholm : «Le concepteur aurait pu faire autrement ». Il suggère donc de renoncer à 
la définition classique de la liberté et à sa révision par l'analyse stratégique au profit de la révision radicale opérée par la philosophie analytique de l'action. Autrement, comment pourrait-on être libre dans le respect à la lettre d'une contrainte?

\subsection{Des stratégies multi-dépendantes}

Les stratégies que nous venons d'étudier (négociation, interprétation, détournement, rejet argumenté, absorption par anticipation, contre-contrainte, phagocytage) sont, à l'évidence, dépendantes de multiples facteurs :

1) L'âge et la personnalité des concepteurs est un facteur décisif (tel architecte a consacré les deux-tiers de l'entretien au rôle de la négociation, tel autre semble utiliser prioritairement les ressources plus discrètes de l'anticipation).

2) La formation est aussi déterminante (les conduites d'application et de rejet argumenté de la contrainte semblent liées aux profils scientifiques, alors que la culture beaux-arts ne semble pas préparer à des actions du même type). Cela n'est pas sans évoquer les travaux de Terry Shinn (1980) sur les rapports entre formation et styles de raisonnement. Notons toutefois que deux architectes ayant un profil scientifique ont porté des jugements opposés sur l'aisance qu'ils éprouvaient face à l'ABF (absence de texte) ou aux services incendie (texte précis); et c'est paradoxalement celui qui avait entrevu une carrière scientifique (père polytechnicien, formation en classes préparatoires) qui s'accommode aujourd'hui le mieux de la négociation. Des études plus précises seraient à faire sur ce point.

3) Les stratégies utilisées dépendent des phases de travail auxquelles elles s'appliquent, non pas parce que le contenu des tâches justifierait telle ou telle attitude, mais parce que les rapports avec telle classe d'acteurs y sont plus fréquents (on négocie plus avec certains interlocuteurs que d'autres). En voici un témoignage :

«Je dirais que les stratégies sont fonction des phases du projet. Il peut y avoir négociation avec le maître d'ouvrage jusqu'au moment où les marchés sont signés. [...] La négociation, on l'a avec les entreprises. Avec l'incendie, très peu. À l'inverse, avec l'ABF, il y a énormément de négociation. »

4) Les stratégies du concepteur dépendent des ressources mobilisées par les architectes. Le pouvoir est la ressource principale. Les stratégies impliquent souvent un usage simultané de 
plusieurs des formes de pouvoir décrites par Crozier et Friedberg (1997 : 83) : (1) maîtrise de compétences spécialisées ; (2) contrôle des relations avec l'environnement ; (3) maîtrise de l'information ; (4) maîtrise des règles organisationnelles. Des ressources matérielles, morales, esthétiques, organisationnelles, etc. diverses se combinent en des formes d'action relativement stables. Bornons-nous ici à mentionner le cas de formes d'argumentation rationnelle qui paraissent développées au-delà du nécessaire dans les situations de conception (cf. supra 5.4). Pourquoi ? Probablement parce que le concepteur sait pouvoir tirer bénéfice du caractère définitif de la preuve rationnelle : elle lui permet de faire évoluer le projet sans risque de retour en arrière.

Ainsi, le rejet argumenté de la contrainte de sol définie à mauvais escient pour le concours de la Bibliothèque d'Alexandrie supposait que Snøhetta : connaisse les attributions spécifiques de chacun des acteurs (4); revendique ses compétences face au programmiste (1); taise la règle sans fondement pour conserver l'avantage sur ses concurrents (3). De même, dans le cas des copropriétaires craignant que le nouveau bâtiment ne fasse de l'ombre à leur immeuble, l'architecte a pu démontrer qu'il était faux de prétendre qu'il porterait une ombre sur l'immeuble. Cette action n'a pu être menée à terme qu'en combinant diverses ressources : matérielles, i.e. un « soleil artificiel » (simulateur de la course solaire durant toute l'année) ; relationnelles, i.e. les contacts avec l'école d'architecture de la même ville qui a prêté le simulateur); professionnelles, i.e. la compétence à se servir du simulateur ; morales, i.e. le fait de l'utiliser avec probité, sans détourner les résultats à son avantage, etc. Mais il reste que ces ressources sont intégrées à une forme d'action stable : l'argumentation rationnelle, ici choisie parce que, sous certaines hypothèses (autrui de bonne foi, ouvert à la discussion, etc.), l'architecte savait cette stratégie efficace pour amener ses contradicteurs à son point de vue.

\section{Conclusions}

\section{Résultats factuels}

Il ressort des exemples étudiés dans cet article que la contrainte naît d'une interaction de l'architecte avec des acteurs spécifiques (Maître d'ouvrage, Services d'urbanisme, Commission de sécurité, etc.) Mais deux cas doivent être distingués : (1) Lorsqu'ils appliquent une injonction positive ou doublement bornée, spécialement sous la forme d'une injonction de disposition, ces acteurs 
exercent une contrainte préjudiciable au travail du concepteur. Elles tendent à annuler son travail de conception, érodent son champ d'expertise, fragilisent son statut professionnel, et entravent la recherche de qualité architecturale qui ne peut voir le jour qu'au travers des relations de confiance (Boudon, 1997) ; (2) Lorsqu'ils appliquent des injonctions de principe, y compris même dans le cas d'une injonction négative, ces acteurs ne font qu'entretenir l'activité de conception en forçant simplement l'architecte à élaborer des réponses originales.

Le résultat factuel de cette observation des stratégies des concepteurs est une clarification contre intuitive de l'aversion bien connue des architectes vis-à-vis des contraintes de conception. Gette aversion ne résulte pas d'une restriction directe de sa marge de liberté mais des effets secondaires induits par les contraintes : elles entravent son activité en le forçant à déterminer lesquelles sont à même de s'exercer dans une situation donnée. Ceci explique les difficultés de l'exercice professionnel :

1) Lorsque les textes sont plus nombreux, le risque d'oubli ou de mésinterprétation des textes croît, et la chance d'une sanction croît en conséquence. La prolifération des textes place le concepteur dans une situation d'incertitude, critique pour le développement du projet. En ce sens, le problème auquel l'architecte est confronté n'est pas celui de la contrainte, mais celui d'une surresponsabilisation des actes de conception.

2) Lorsque les textes sont plus nombreux, le budget-temps consacré à l'étude des textes croît, et celui consacré à l'élaboration des propositions architecturales décroît en conséquence. La prolifération des textes met le concepteur en situation de devoir rendre des propositions moins élaborées, ce qui induit une baisse tendancielle de la qualité architecturale. En ce sens, le problème qu'affronte l'architecte n'est pas celui de la contrainte, mais celui de la sous-faisabilité des études de conception ${ }^{26}$.

${ }^{26}$ Ces deux diagnostics peuvent être référés au jugement de certains architectes sur les conditions d'exercice de la profession. L'objectif de faisabilité explique leur préférence pour les contraintes non négociables et leur hantise des révisions du projet : «Avec les services incendie, il y a peu de négociation. Je dirais que c'est plus facile parce que, en fait, il y a un texte qui est écrit, qui est très clair, et la marge de négociation est quasiment nulle. Quand ils te disent "Il nous faut $25 \mathrm{~m}$ entre l'issue de secours et la porte de l'appartement" il y a $25 \mathrm{~m}$. Tu prends le cutch et tu mesures ! Terminé. - Cela ne soulève pas des questions de gestion des projets ? - Ah mais complètement! Sur des gros projets, le maître d'ouvrage ne va pas attendre six mois qu'on ait fini une négociation »; «À chaque fois on retrouve les mêmes problèmes, mais [les check-lists] permettent de ne pas 


\section{Résultats théoriques}

Cet article nous engage également à redéfinir la contrainte. Partons d’une expérience de pensée. Le cumul des contraintes n'étant pas exclu²7, il est aisé d'obtenir les portraits-types d'un projet contraignant et d'un projet peu contraignant. Par exemple, la conception d'un hôpital universitaire en milieu urbain est contraignante : c'est une commande publique (action du contrôleur technique au titre du respect des normes DTU), un lieu de travail (action de l'Inspection du travail, au titre de l'hygiène et de la sécurité), un immeuble de plus de 10 étages (action de la Commission de sécurité et du contrôleur technique), possédant un auditorium (action d'un acousticien), requérant, pourquoi pas, une étude parasismique (action d'un BET) et situé par hypothèse dans le champ de visibilité d'un monument (action de l'architecte des Bâtiments de France). Ce projet définit un cadre d'action plus coercitif que la conception d'un hangar situé sur le territoire d'une commune sans plan local d'urbanisme. Mais pourquoi beaucoup d'architectes préféreraient-ils se voir confier l'étude de l'hôpital ? Le montant global des travaux n'est pas la seule explication. Ce paradoxe résulte du préjugé selon lequel la liberté est définie par l'absence de contrainte. Si l'architecte exprime régulièrement sa préférence pour des situations contraignantes, c'est que la liberté du concepteur ne varie pas en raison inverse des contraintes qui s'exercent sur lui.

Les sociologues doivent être attentifs au fait que la position de bon sens, que l'on trouvait dans la philosophie morale d'un Moritz Schlick, doit être révisée plus profondément que ne l'a proposé l'analyse stratégique. Comme nous l'avons vu dans cet article, l'idée même d'un rapport négatif entre liberté et contrainte doit être abandonnée : elle viole l'échelle de perception

oublier certains points et de ne pas se dire, une fois le projet ficelé, “On a oublié ce truc là !" Parfois, ça peut avoir des conséquences graves... Avant de commencer à dessiner, il faut recueillir un maximum de données pour ne pas se demander au moment du permis : "On est dans quelle catégorie incendie ? Ah oui, $\mathrm{R}+7$, alors il nous faut une voie échelle. Et la voie échelle on la met où ? On n’a plus de place !" Et alors on redessine tout ! Là, c'est vraiment une question de rentabilité et d'efficacité du travail. » Les objectifs sont toujours les mêmes : ne pas perdre de temps, ne pas bloquer le projet, ne pas remettre en question ce qui est déjà dessiné. Ce sont là les axiomes fondamentaux de la conduite des projets et ils exercent une contrainte autrement plus forte que les contraintes qui orientent la conception : c'est une question de survie de l'agence.

${ }^{27}$ Conan (1995) raconte les péripéties d'une opération immobilière dans laquelle on voit l'architecte se 
de la contrainte entre une interdiction et une prescription ; elle est impuissante à expliquer les cas de phagocytage, dans lesquels les architectes parviennent à se dégager de la contrainte en l'appliquant à la lettre ou par excès (supra, 5. 7).

\section{Appendice sur la logique déontique}

La propriété selon laquelle toute contrainte possède nécessairement un format conditionnel (fut-il sous-entendu) trouve aussi une application en « logique déontique », qui formalise souvent ses énoncés sans tenir compte du caractère conditionnel des injonctions. Rappelons ici les notations introduites par Georg Henrik von Wright (1963) : [p] désignant un état du monde, on peut distinguer quatre types de transformations $[\mathrm{T}]:(\mathrm{pTp}),(\sim \mathrm{pTp}),(\mathrm{pT} \sim \mathrm{p}),(\sim \mathrm{pT} \sim \mathrm{p})$. L'action est formalisée par le couple : [d] faire [doing], [f] s'abstenir [forbearing]. Supposons par exemple que p symbolise l'état « fenêtre ouverte ». L'action de ne pas ouvrir la fenêtre peut s'écrire : f( pTp). Ces notations permettent de traiter les états et les actions portant sur les états du monde.

L'auteur introduit ensuite les opérateurs déontiques $[\mathrm{O}]$ obligation et $[\mathrm{P}]$ permission, en vue de qualifier les normes d'action. Je peux maintenant interdire à autrui d'ouvrir la fenêtre : $\mathrm{Of}(\sim \mathrm{p} T \mathrm{p})$; l'obliger à ouvrir la fenêtre : $\operatorname{Od}(\sim p T p)$; lui permettre d'ouvrir la fenêtre : $\operatorname{Pd}(\sim p T p)$; ou lui permettre de ne pas ouvrir la fenêtre : $\operatorname{Pf}(\sim p T p)$.

En dépit de l'intérêt de cette notation, il semble difficile de traduire les contraintes de conception en logique déontique, du fait que l'opérateur déontique $[\mathrm{P}]$ ne se rattache pas au format conditionnel général. Examinons quelques conditionnelles données par von Wright. La phrase : "Si le chien aboie, ne cours pas" est qualifiée d'interdiction (von Wright, 1963 : 71). Sur le même modèle : "Si le chien aboie, cours" exprimerait une obligation. Plus loin, von Wright prétend que les phrases impératives expriment parfois des permissions et non pa seulement des obligations ou des interdictions (1963: 90). «J'arrive au coin d'une rue et les feux disent "traversez maintenant", la norme (prescription) qui m'est adressée par ces mots est une permission de traverser et non pas une commande de le faire » (1963 : 90). Traduisons ces phrases au format conditionnel :

\footnotetext{
Si le chien aboie, alors ne cours pas (interdiction)

Si le chien aboie, alors cours (ordre)
} 
[Si tu le veux], alors traverse maintenant (permission)

Une fois ces phrases réduites au même format, on constate - contrairement à l'habitude que nous avons de juger un ordre plus contraignant qu'une permission - que l'un et l'autre présentent parfois le même degré de contrainte. Car, dans le cas d'une permission, dès que la condition exprimée par le premier membre est satisfaite [si le piéton veut traverser], il est obligé de traverser lorsque le signal s'allume s'il ne veut pas se faire écraser. Cette contrainte s'exerce, non seulement dans le cas la volonté du piéton se fonde sur une obligation extérieure - Si tu dois te rendre au travail, alors traverse maintenant - mais aussi dans le cas où il se promène librement. Lorsque la décision de traverser la rue est prise, le signal "traversez maintenant" exerce une contrainte sur lui, ce dont ne rend pas compte l'analyse déontique de la permission.

Selon la logique déontique de von Wright, si p désigne "être de l'autre côté de la rue", la phrase "traversez maintenant" s'interprète $\operatorname{Pd}(\sim \mathrm{pT})$. Or le format conditionnel que nous avons introduit transforme la permission en obligation. « Si tu veux traverser, alors traverse maintenant » exprime l'obligation $\operatorname{Od}(\sim p T p)$. Que cette traduction soit possible montre qu'ici la permission équivaut à une obligation conditionnelle.

\section{Références}

Accarias, G. (1879). Précis de droit romain, contenant l'exposé des principes généraux, le texte, la traduction et l'explication des Institutes de fustinien... tome 1. Paris, A. Cotillon et Cie éditeurs.

Boudon, Ph. et al. (1994). Enseigner la conception architecturale. Paris, Éditions de la Villette.

Boudon, R., éd. (1992). Traité de sociologie. Paris, PUF.

Boudon, R. (1997). Faire confiance plutôt qu'interdire ou ordonner, Sociétal, 12, 49-50.

Boudon, R. (2003). Raison, bonnes raisons. Paris, PUF.

Champy, F. (1999). Vers la déprofessionalisation. L'évolution des compétences des architectes en France depuis les années 80. Cahiers de la Recherche architecturale et urbaine, 2-3, 27-38.

Chazel, F. (1990). Normes et valeurs sociales. Encyclopedia universalis, 16, 450-453.

Chazel, F. (1992). Pouvoir. Boudon, R. dir., Traité de sociologie. Paris, PUF, 195-226.

Chisholm, R.M. (1997). La liberté humaine et le moi. Neuberg, M. éd. La responsabilité. Questions philosophiques. Paris, PUF, 39-54 [1964].

Code de la construction et de l'habitation (2000). Commenté par P. Cornille et A. Durance. Paris, Litec.

Code de l'urbanisme (2000). Commenté et annoté par F. Bouyssou et J. Hugot. Paris, Litec. 
Conan, M. (1995). Les jeux imbriqués de la conception architecturale. Prost, R. éd. Concevoir, inventer, créer. Paris, L’Harmattan, 153-188.

Crozier, M. et Friedberg, E. (1977). L'acteur et le système. Les contraintes de l'action collective. Paris, Éditions du Seuil.

Farel, A. (1995). Conception d'un bâtiment : l'organisation d'un travail collectif. Prost, R. éd. Concevoir, inventer, créer. Paris, L'Harmattan, 51-63.

Friedberg, E. (1992). Les quatre dimensions de l'action organisée, Revue française de Sociologie, 33 (4), $531-557$.

Friedberg, E. (1993). Le pouvoir et la règle. Dynamique de l'action organisée. Paris, Éditions du Seuil.

Goulet, P. (1983). Kazuo Shinohara : maison sous une ligne à haute tension. Architecture d'Aujourd'hui, 226, 41-43.

Guadet, J. (1895). Code des devoirs professionnels de l'architecte présenté au nom de la Société centrale des Architectes français, L'Architecture, 33, 288-289.

Guesnard, E. (1999). Deux monolithes, Architecture d'Aujourd'hui, 320, 62-63.

Melden, A.I. (1961). Free action. London, Routledge \& Keagan Paul.

Michelin, N. (2003). Profession : SHONSU et SHOBSDO font-ils œuvre utile ? AMC, 135, 30-31.

Montlibert, C. de (1995). L'impossible autonomie de l'architecte. Sociologie de la production architecturale. Strasbourg, Presses universitaires de Strasbourg.

Moulin, R. et al. (1973). Les architectes. Métamorphoses d'une profession libérale. Paris, Calmann-Lévy.

Moulin, R. et Lautman, J. (1978). Les architectes. La profession libérale et son aggiornamento. Cahiers de la Recherche architecturale, 2, 49-55.

Neuberg M. (1990). La contrainte, Dialogue, 29, 491-522.

Neuberg, M. éd. (1991). Théorie de l'action. Textes majeurs de la philosophie analytique de l'action. Liège, Mardaga.

Nouvel, J., G. Lezénés, P. Soria, Architecture Studio (1995). Institut du Monde arabe. Paris, Les Éditions du Demi-Cercle.

Nozick, R. (1991). La contrainte. Neuberg, M. éd., Théorie de l'action. Textes majeurs de la philosophie analytique de l'action, Liège, Mardaga, 271-310 [1969].

Oppenheim, F. (1961). Dimensions of freedom. New York, St Martin's Press.

Prost, R. éd. (1995). Concevoir, inventer, créer. Réflexions sur les pratiques. Paris, L'Harmattan. 
Prost, R. (1999). Les pratiques architecturales en mutation. Cahiers de la Recherche architecturale et urbaine, 2-3, 85-94.

Raynaud, D. (1998). Architectures comparées. Marseille, Parenthèses.

Raynaud, D. (1999). Le schème, opérateur de la conception architecturale. Intellectica, 29, 35-69.

Raynaud, D. (2001). Compétences et expertise professionnelle de l'architecte dans le travail de conception, Sociologie du Travail, 43 : 451-469.

Raynaud, D. (2002). Cinq essais sur l'architecture. Étude sur la conception de projets de l'Atelier Zố, Scarpa, Le Corbusier, Pei. Paris, L'Harmattan.

Schlick, M. (1997). Quand sommes-nous responsables ? Neuberg, M. éd., La responsabilité. Questions philosophiques. Paris, PUF, 27-38 [1930].

Shinn, T. (1980). Division du savoir et spécificité organisationnelle, Revue française de Sociologie, 21 : 3-35.

Simon, H.A. (1969). Sciences des systèmes, sciences de l'artificiel. Paris, Epi.

Tapie, G. (1999). Professions et pratiques. Cahiers de la Recherche architecturale et urbaine, 2-3, 65-74.

Thuderoz, G. (2000). Négociations : essai de sociologie du lien social. Paris, PUF.

Toussaint, J.Y. et Younès, G. éds. (1997). Architectes, ingénieurs : des métiers et des professions. Paris, Éditions de la Villette.

Weber, M. (1971). Économie et société, 1 : Les catégories de la sociologie. Paris, Plon.

Wittgenstein, L. (1961). Tractatus philosophicus. Investigations philosophiques. Paris, Gallimard.

Wright, G.H. von (1963). Norm and action. A logical enquiry, London, Routledge and Kegan Paul. 